\title{
Solutions to the Optical Cascading Equations
}

\author{
S. LAFortune and P. Winternitz \\ Centre de Recherches Mathématiques, \\ Université de Montréal, \\ C. P. 6128, Succ. Centre-ville, \\ Montréal, (QC) H3C 3J7, \\ Canada \\ C.R. Menyuk \\ Departement of Electrical Engineering \\ University of Maryland \\ Baltimore, Maryland \\ 21228-5398
}

Group theoretical methods are used to study the equations describing $\chi^{(2)}: \chi^{(2)}$ cascading. The equations are shown not to be integrable by inverse scattering techniques. On the other hand, these equations do share some of the nice properties of soliton equations. Large families of explicit analytical solutions are obtained in terms of elliptic functions. In special cases these periodic solutions reduce to localized ones, i.e. solitary waves. All previously known explicit solutions are recovered and many new ones are obtained. 42.65.-k, 42.65.Tg, 03.40.Kf, 02.20.-a 


\section{INTRODUCTION}

Materials with a significant $\chi^{(3)}$ nonlinearity exhibit solitonlike beam or pulse propagation. These materials can be modeled by the nonlinear Schrödinger equation and its variants [1] which are known to be integrable and possess exact soliton solutions.

In the case of $\chi^{(2)}$ materials, it has been possible to produce solitary waves through $\chi^{(2)}: \chi^{(2)}$ cascading [2]. Moreover, several particular cases of solitary wave solutions of the system describing this phenomena have been obtained numerically and analytically ([3-9]).

The solitons of $\chi^{(3)}$ type are known to belong to families that include periodic solutions. It is essential to know if this is the case for the solitary waves of the $\chi^{(2)}$ type, mainly because the system describing them is not integrable. We do not know what properties of integrable systems these solitary wave solutions possess.

Moreover, the periodic solutions containing these solitary waves as limiting cases can be important in their own right, specially if they are stable. For instance they may propagate as a background to solitary wave signals.

In this paper we find families of periodic solutions expressed in terms of elliptic functions. As special limiting cases we obtain elementary trigonometric solutions and also localized solitary waves (including all those found earlier). We mention that not all of the solitary waves are stable [10-12] and that the stability of periodic solutions needs a separate study.

In Section 2 we present the $\chi^{(2)}: \chi^{(2)}$ cascading equations and find their Lie point symmetry group. We then reduce the system to a set of four coupled real ordinary differential equations for traveling wave solutions. These reduced equations are further studied in Section 3. We show that in general the equations do not have the Painlevé property [13-14] so the cascading equations are not integrable. We do however show that well behaved analytical solutions exist in special cases. Explicitly, elliptic function solutions are presented in Section 4 together with their elementary function limits. Some further special cases are discussed in Section 5. Conclusions are drawn in the final Section 6. 


\section{THE CASCADING EQUATIONS AND THEIR REDUCTION}

Let us write the cascading equations in the normalized form [3]

$$
\begin{gathered}
i a_{1 t}-\frac{r}{2} a_{1 x x}+a_{1}^{*} a_{2}=0, \\
i a_{2 t}-\beta a_{2}-i \delta a_{2 x}-\frac{\alpha}{2} a_{2 x x}+a_{1}^{2}=0,
\end{gathered}
$$

where $a_{1}$ and $a_{2}$ are the (complex) envelopes of the fundamental and second harmonic wave, respectively. They are functions of two variables, namely $t$, the normalized distance along the wave guide and $x$, the normalized transverse coordinate. The constants involved are $r= \pm 1$ and $\alpha, \beta, \delta$ (all reals).

To obtain explicit solutions we use the method of symmetry reduction, i.e. use Lie point symmetries to reduce the equations (2.1) to ordinary differential equations. We first replace eq.(2.1) by four real equations, putting

$$
\begin{gathered}
a_{k}(x, t)=R_{k}(x, t) e^{i \phi_{k}(x, t)}, k=1,2, \\
0 \leq R_{k}<\infty, \quad 0 \leq \phi_{k}<2 \pi,
\end{gathered}
$$

and apply a standard algorithm to find their symmetry group $[15,16,17]$. In the generic case, when all constants in the equation are arbitrary, the symmetry group of the equations is just three-dimensional, consisting of translations in $x$ and $t$ and corelated shifts of the phase $\phi_{1} \rightarrow \phi_{1}+\lambda, \phi_{2} \rightarrow \phi_{2}+2 \lambda$. The Lie algebra of the symmetry group has a basis consisting of the operators

$$
P_{1}=\partial_{x}, \quad P_{0}=\partial_{t}, \quad W=\partial_{\phi_{1}}+2 \partial_{\phi_{2}}
$$

We shall consider solutions invariant under the subgroup generated by

$$
X=v P_{1}+\omega P_{0}+\left(\kappa_{1} \omega-\omega_{1} v\right) W
$$

where $v, \omega, \kappa_{1}$ and $\omega_{1}$ are real constants.

The invariant solutions then have the form 


$$
\begin{gathered}
a_{1}(\xi)=R_{1}(\xi) e^{i \phi_{1}(\xi)} e^{i\left(\kappa_{1} t-\omega_{1} x\right)}, \\
a_{2}(\xi)=R_{2}(\xi) e^{i \phi_{2}(\xi)} e^{2 i\left(\kappa_{1} t-\omega_{1} x\right)}, \\
\xi=\omega x-v t .
\end{gathered}
$$

The four real functions $R_{1}, R_{2}, \phi_{1}, \phi_{2}$ satisfy the following system of coupled ordinary differential equations

$$
\begin{gathered}
-\frac{r}{2} \omega^{2}\left(2 R_{1}^{\prime} \phi_{1}^{\prime}+R_{1} \phi_{1}^{\prime \prime}\right)+\left(r \omega \omega_{1}-v\right) R_{1}^{\prime}+R_{1} R_{2} \sin \phi=0, \\
-\frac{\alpha}{2} \omega^{2}\left(2 R_{2}^{\prime} \phi_{2}^{\prime}+R_{2} \phi_{2}^{\prime \prime}\right)+\left(2 \alpha \omega \omega_{1}-v-\delta \omega\right) R_{2}^{\prime}-R_{1}^{2} \sin \phi=0 \\
-\frac{r}{2} \omega^{2}\left(R_{1}^{\prime \prime}-R_{1} \phi_{1}^{\prime 2}\right)+\left(v-r \omega \omega_{1}\right) R_{1} \phi_{1}^{\prime}+\left(\frac{r}{2} \omega_{1}^{2}-\kappa_{1}\right) R_{1} \\
+R_{1} R_{2} \cos \phi=0 \\
-\frac{\alpha}{2} \omega^{2}\left(R_{2}^{\prime \prime}-R_{2} \phi_{2}^{\prime 2}\right)-\left(2 \alpha \omega \omega_{1}-v-\delta \omega\right) R_{2} \phi_{2}^{\prime} \\
+\left(2 \alpha \omega_{1}^{2}-2 \kappa_{1}-\beta-2 \delta \omega_{1}\right) R_{2}+R_{1}^{2} \cos \phi=0 \\
\phi \equiv \phi_{2}-2 \phi_{1} .
\end{gathered}
$$

It is the system of equations (2.6)-(2.9) that we wish to solve.

\section{ANALYSIS OF THE REDUCED EQUATIONS}

\section{A. Phase locked solutions}

The system $(2.6), \ldots,(2.9)$ is in general quite difficult to decouple and solve. It is greatly simplified if we impose a supplementary restriction on the phases, namely

$$
\phi=k \pi, \quad k \in \mathbb{Z}, \text { i.e. } \sin \phi=0, \cos \phi=\epsilon, \epsilon^{2}=1 .
$$

We shall call such solutions "phase locked solutions". Let us first assume

$$
r \alpha \omega \neq 0
$$

and simplify notations, putting 


$$
\begin{array}{cc}
A=\frac{r \omega \omega_{1}-v}{r \omega^{2}}, & B=\frac{2 \alpha \omega \omega_{1}-v-\delta \omega}{\alpha \omega^{2}}, \\
C=2 \frac{2 \alpha \omega_{1}^{2}-2 \kappa_{1}-2 \delta \omega_{1}-\beta}{\alpha \omega^{2}}, D & =\frac{r \omega_{1}^{2}-2 \kappa_{1}}{r \omega^{2}} \\
M_{1}=-\frac{2 N_{1}}{r \omega^{2}}, & M_{2}=-\frac{2 N_{2}}{\alpha \omega^{2}} .
\end{array}
$$

The equations $(2.6), \ldots,(2.9)$ can be rewritten as

$$
\begin{gathered}
\phi_{1}^{\prime}=A+\frac{M_{1}}{R_{1}^{2}} \\
\phi_{2}^{\prime}=B+\frac{M_{2}}{R_{2}^{2}}, \\
R_{1}^{\prime \prime}-R_{1} \phi_{1}^{\prime 2}+2 A R_{1} \phi_{1}^{\prime}-D R_{1}-\frac{2 \epsilon}{r \omega^{2}} R_{1} R_{2}=0 \\
R_{2}^{\prime \prime}-R_{2} \phi_{2}^{\prime 2}+2 B R_{2} \phi_{2}^{\prime}-C R_{2}-\frac{2 \epsilon}{\alpha \omega^{2}} R_{1}^{2}=0 .
\end{gathered}
$$

\section{B. Case $M_{1} M_{2} \neq 0$}

The phase locking condition (3.1) imposes a relation between $R_{1}$ and $R_{2}$, namely

$$
B+\frac{M_{2}}{R_{2}^{2}}=2 A+\frac{2 M_{1}}{R_{1}^{2}}
$$

so that the system $(3.4), \ldots,(3.8)$ is overdetermined. Expressing $R_{1}, \phi_{1}^{\prime}$ and $\phi_{2}^{\prime}$ in terms of $R_{2}$ and substituing into (3.6) and (3.8), we obtain two second order ordinary differential equations for $R_{2}$. These turn out to be compatible only for $R_{1}$ and $R_{2}$ constant. This case will be considered separately in Section 5 below.

\section{Case $M_{1} M_{2}=0$}

Again $R_{1}$ and $R_{2}$ are constant, unless we have

$$
M_{1}=M_{2}=0
$$

Let us investigate case (3.9). We have 


$$
\phi_{1}^{\prime}=A, \quad \phi_{2}^{\prime}=B=2 A .
$$

We put

$$
A_{0}=A^{2}-D, \quad B_{0}=-\frac{2 \epsilon}{r \omega^{2}}, \quad C_{0}=B^{2}-C, \quad D_{0}=-\frac{2 \epsilon}{\alpha \omega^{2}},
$$

and obtain a system of two ordinary differential equations

$$
\begin{aligned}
& R_{1}^{\prime \prime}+A_{0} R_{1}+B_{0} R_{1} R_{2}=0, \\
& R_{2}^{\prime \prime}+C_{0} R_{2}+D_{0} R_{1}^{2}=0 .
\end{aligned}
$$

This system is not overdetermined since the constraint (3.8) is now simply the condition (3.10) on the constants, i.e.

$$
2 \alpha v-r v-\delta r \omega=0
$$

Eliminating $R_{2}$ from eq.(3.12), we obtain a fourth order equation for $R_{1}$, namely

$$
\left(\frac{R_{1}^{\prime \prime}}{R_{1}}\right)^{\prime \prime}+C_{0} \frac{R_{1}^{\prime \prime}}{R_{1}}-B_{0} D_{0} R_{1}^{2}+A_{0} C_{0}=0 .
$$

If the original equations (2.1) are integrable then eq.(3.14) should have the Painlevé property, i.e. have no movable singularities other than poles [13,14]. An algorithmic test exists $[13,18]$ establishing certain properties of an equation, necessary for it to have the Painlevé property.

Thus, the general solution of eq.(3.14) must allow an expansion in the neighbourhood of any singular point of the form

$$
R_{1}=\sum_{k=0}^{\infty} a_{k}\left(\xi-\xi_{0}\right)^{k+p}
$$

with $p$ a negative integer, $a_{0} \neq 0$ and three of the coefficients $a_{k}$ arbitrary. Then $R_{1}$ has a good chance of representing the general solution of eq.(3.14), depending on four arbitrary constants (one of them being $\xi_{0}$, the position of the pole). The values of $k$ for which $a_{k}$ are arbitrary (i.e. are not determined by a recursion relation), are called "resonance" values.

Substituting the expansion (3.15) into eq.(3.14), we find $p=-2, a_{0}^{2}=36 /\left(B_{0} D_{0}\right)$ and the resonance values 


$$
r=-1,6,(5 \pm i \sqrt{23}) / 2 .
$$

Thus, we have only one nonnegative integer, namely $r=6$, rather than the three ones needed. An analysis of the obtained recursion relations shows that $a_{0}, \ldots, a_{5}$ are fully determined, $a_{6}$ is indeed free and can be chosen arbitrarily. Then $a_{7}$ and all the higher terms are fully determined in terms of $\xi_{0}$ and $a_{6}$ (and of course $A_{0}, B_{0}, C_{0}$ and $D_{0}$ ).

Thus, eq.(3.14) does not have the Painlevé property and the cascading equations (2.1) are not integrable.

The Painlevé analysis does however indicates that families of "well behaved" solutions should exist (i.e. single valued in the neighbourhood of their movable singularities), depending on one or two free parameters rather than on four. We shall find such solutions below.

An alternative procedure is to solve eq.(3.12), again under the condition (3.13), for $R_{2}$. We obtain the ordinary differential equation

$$
\begin{array}{r}
2\left(R_{2}^{\prime \prime}+C_{0} R_{2}\right)\left(R_{2}^{\prime \prime \prime \prime}+C_{0} R_{2}^{\prime \prime}\right)-\left(R_{2}^{\prime \prime \prime}+C_{0} R_{2}^{\prime}\right)^{2} \\
+4\left(A_{0}+B_{0} R_{2}\right)\left(R_{2}^{\prime \prime}+C_{0} R_{2}\right)^{2}=0 .
\end{array}
$$

A Painlevé analysis of eq.(3.17) leads to the same conclusion as that of eq.(3.14).

\section{Introduction of the Elliptic Function Equation}

Let us look for solutions $R_{2}(\xi)$ satisfying eq.(3.17) and also the elliptic function equation

$$
R_{2}^{\prime 2}=\gamma_{4} R_{2}^{4}+\gamma_{3} R_{2}^{3}+\gamma_{2} R_{2}^{2}+\gamma_{1} R_{2}+\gamma_{0} .
$$

The compatibility of eq.(3.18) and (3.17) implies

$$
\gamma_{4}=0
$$

and imposes six relations between the constants in (3.18) and (3.17). These allow for the following solutions. 


$$
\text { 1. } \gamma_{3} \neq 0, \quad C_{0}\left(C_{0}-A_{0}\right) \neq 0 \text {. }
$$

The constants $\gamma_{\mu}$ in eq.(3.18) are completely specified:

$$
\begin{aligned}
\gamma_{3}=-\frac{2 B_{0}}{3}, \gamma_{2} & =C_{0}-2 A_{0}, \quad \gamma_{1}=\frac{\left(C_{0}+2 A_{0}\right)\left(C_{0}-A_{0}\right)}{B_{0}}, \\
\gamma_{0} & =\frac{\left(C_{0}+2 A_{0}\right)^{2}\left(C_{0}-A_{0}\right)}{6 B_{0}^{2}} .
\end{aligned}
$$

The other amplitude, $R_{1}(\xi)$ is given directly by the expression

$$
R_{1}^{2}=\frac{1}{D_{0}}\left(B_{0} R_{2}^{2}+2\left(A_{0}-C_{0}\right) R_{2}+\frac{\left(A_{0}-C_{0}\right)\left(2 A_{0}+C_{0}\right)}{2 B_{0}}\right)
$$

and we must require $R_{1}^{2}$ to satisfy

$$
R_{1}^{2} \geq 0
$$

in the entire range of values of $R_{2}$, a condition to be analysed below.

Notice that eq.(3.18) will have solutions depending on just one parameter, an integration constant, since the coefficients $\gamma_{\mu}$ are fixed in terms of $A_{0}, B_{0}, C_{0}$ and $D_{0}$. The only constraints on the constants in the original equations (2.1) $r, \alpha, \beta$ and $\delta$ and those introduced in the reduction procedure (2.5) namely $v, \omega, \kappa_{1}$ and $\omega_{1}$ are (3.2), (3.13) and also (3.23).

$$
\text { 2. } \gamma_{3} \neq 0, \quad C_{0}=0 \text {. }
$$

In this case we obtain

$$
\gamma_{3}=-\frac{2 B_{0}}{3}, \quad \gamma_{2}=-2 A_{0}, \quad \gamma_{1}=-\frac{2 A_{0}^{2}}{B_{0}}
$$

and $\gamma_{0}$ is arbitrary. Moreover, we have

$$
R_{1}=\sqrt{\frac{B_{0}}{D_{0}}}\left(R_{2}+\frac{A_{0}}{B_{0}}\right) .
$$

Thus, we obtain a two parameter family of solutions, the parameters being $\gamma_{0}$ and a constant arising in the integration of eq.(3.18). The constraints on the coefficients are (3.2), (3.13) and $C_{0}=0$, i.e.

$$
2 \alpha v^{2}+\omega\left(2 \kappa_{1} \omega+\beta \omega-2 \omega_{1} v\right)=0
$$


(we have $r^{2}=1$ ).

$$
\text { 3. } \gamma_{3} \neq 0, C_{0}=A_{0} \text {. }
$$

We then have

$$
\gamma_{3}=-\frac{2 B_{0}}{3}, \gamma_{2}=-A_{0}, \quad \gamma_{1}=0
$$

and $\gamma_{0}$ arbitrary. Again, (3.18) provides a two parameter family of solutions and the constraint $C_{0}=A_{0}$ is

$$
3 \alpha v^{2}+2 r \omega(\alpha-2 r)\left(\omega_{1} v-\kappa_{1} \omega\right)+2 \beta \omega^{2}=0
$$

and we have

$$
R_{1}=\sqrt{\frac{B_{0}}{D_{0}}} R_{2} .
$$

4. $\gamma_{3}=0$.

In this case eq.(3.18) reduces to

$$
R_{2}^{\prime 2}=-C_{0} R_{2}+\gamma_{0}
$$

The solutions are

$$
\begin{gathered}
R_{1}=0 \\
R_{2}= \begin{cases}-\frac{C_{0}}{4}\left(\xi-\xi_{0}\right)^{2}+\mu & C_{0} \neq 0 \\
\mu\left(\xi-\xi_{0}\right) & C_{0}=0 .\end{cases}
\end{gathered}
$$

\section{E. Phase Locked Solutions for $\alpha=0$}

We return to eq.(2.6),..,(2.9) for $\alpha=0, \sin \phi=0, \cos \phi=\epsilon$. From eq.(2.7) we see that $R_{2} \neq$ const implies 


$$
v+\delta \omega=0
$$

Eq.(2.6) can be integrated to give

$$
\phi_{1}^{\prime}=\frac{2 N}{r \omega^{2}} \frac{1}{R_{1}^{2}}+\frac{r \omega \omega_{1}-v}{2},
$$

where $N$ is an integration constant.

In order to have $R_{1} \neq 0$ we impose

$$
2 \kappa_{1}+\beta+2 \delta \omega_{1} \neq 0
$$

and obtain from eq.(2.9) that we have

$$
R_{1}=\left[\epsilon\left(2 \kappa_{1}+\beta+2 \delta \omega_{1}\right) R_{2}\right]^{1 / 2} .
$$

Finally, eq.(2.8) implies that $R_{2}$ satisfies the elliptic function equation

$$
\left(R_{1}^{\prime}\right)^{2}=-2 B_{0} R_{2}^{3}-4 A_{0} R_{2}^{2}+S R_{2}-\frac{16 N^{2}}{\omega^{4}\left(2 \kappa_{1}+\beta+2 \delta \omega_{1}\right)^{2}} .
$$

Since both $N$ and $S$ are arbitrary integration constants, eq.(3.40) yields a 3 parameter family of solutions.

\section{SOLUTIONS IN TERMS OF ELLIPTIC FUNCTIONS AND THEIR LIMITING CASES}

\section{A. General Comments on the Elliptic Function Equation}

In Section 3 we have obtained three equations of the type

$$
R^{\prime 2}=\beta_{3} R^{3}+\beta_{2} R^{2}+\beta_{1} R+\beta_{0}
$$

where $\beta_{i}$ are real constants and $\beta_{3} \neq 0$. Putting

$$
f=\beta_{3} R
$$

we obtain the equation 


$$
f^{\prime 2}=f^{3}+\beta_{2} f^{2}+\beta_{1} \beta_{3} f+\beta_{0} \beta_{3}^{2}
$$

We introduce the roots $f_{1}, f_{2}$ and $f_{3}$ of the polynomial on the right hand side of eq.(4.3) and rewrite this equation as

$$
f^{\prime 2}=\left(f-f_{1}\right)\left(f-f_{2}\right)\left(f-f_{3}\right)
$$

The solutions of this equation can be expressed in terms of Jacobi elliptic functions [19] if the three roots are distinct. The case of multiple roots leads to solutions in terms of elementary functions.

Let us first consider the case of three real roots and order them to satisfy $f_{1} \leq f_{2} \leq f_{3}$. We are only interested in real solutions.

1. $f_{1} \leq f \leq f_{2}<f_{3}$

We obtain a finite periodic solution

$$
\begin{gathered}
f=\left(f_{2}-f_{1}\right) \operatorname{sn}^{2}(\mathrm{u}, \mathrm{k})+\mathrm{f}_{1}, \\
k^{2}=\frac{f_{2}-f_{1}}{f_{3}-f_{1}}, \quad u=\frac{\sqrt{f_{3}-f_{1}}}{2}\left(\xi-\xi_{0}\right),
\end{gathered}
$$

where $\xi_{0}$ is a real integration constant.

$$
\text { 2. } f_{1} \leq f \leq f_{2}=f_{3}
$$

A special (limiting) case of solution (4.5) is obtained for $f_{2}=f_{3}$, namely the solitary wave solution

$$
f=\left(f_{2}-f_{1}\right) \tanh ^{2}(\mathrm{u})+\mathrm{f}_{1}
$$

with $u$ as in equation (4.5).

$$
\text { 3. } f_{1}<f_{2}<f_{3} \leq f
$$

We obtain a singular periodic solution

$$
f=\left(f_{3}-f_{1}\right) \frac{1}{\mathrm{sn}^{2}(\mathrm{u}, \mathrm{k})}+f_{1},
$$


with $u$ and $k$ as in eq.(4.5).

$$
\text { 4. } f_{1}=f_{2}<f_{3} \leq f
$$

For $f_{1}=f_{2}$ solution (4.7) reduces to an elementary periodic singular solution namely

$$
f=\left(f_{3}-f_{1}\right) \frac{1}{\sin ^{2}(u)}+f_{1} .
$$

5. $f_{1}<f_{2}=f_{3} \leq f$

For $f_{2}=f_{3}$ solution (4.7) reduces to a "singular solitary wave", namely

$$
f=\left(f_{3}-f_{1}\right) \operatorname{cotanh}^{2}(\mathrm{u})+\mathrm{f}_{1} .
$$

6. $f_{1}=f_{2}=f_{3} \leq f$

A triple root corresponds to a "singular algebraic solitary wave"

$$
f=\frac{4}{\left(\xi-\xi_{0}\right)^{2}}+f_{1} .
$$

The case of one real and two mutually complex conjugated roots leads to singular periodic solutions.

$$
\text { 7. } f_{1}=a+i b, \quad f_{2}=a-i b, \quad f_{3}, a, b \in \mathbb{R}, b>0
$$

The solution is

$$
\begin{gathered}
f=\frac{f_{3}+P+\left(f_{3}-P\right) \mathrm{cn}(\mathrm{u}, \mathrm{k})}{1+\mathrm{cn}(\mathrm{u}, \mathrm{k})}, \\
u=\sqrt{P}\left(\xi-\xi_{0}\right), \quad k^{2}=\frac{P+a-f_{3}}{2 P}, \quad P^{2}=\left(a-f_{3}\right)^{2}+b^{2} .
\end{gathered}
$$

In the limit $b \rightarrow 0$ solution (4.11) reduces to the solitary wave (4.6).

\section{B. Explicit Solutions of the Cascading Equations for $\alpha \neq 0$}

1. The case (3.20) 
We have

$$
\begin{gathered}
\beta_{2}=C_{0}-2 A_{0}, \quad \beta_{1} \beta_{3}=-\frac{2}{3}\left(C_{0}+2 A_{0}\right)\left(C_{0}-A_{0}\right) \\
\beta_{0} \beta_{3}^{2}=\frac{2}{27}\left(C_{0}+2 A_{0}\right)^{2}\left(C_{0}-A_{0}\right), \quad C_{0} \neq 0, \quad C_{0} \neq A_{0}
\end{gathered}
$$

in eq.(4.3). Using eq.(3.22) we have

$$
R_{2}=-\frac{3}{2 B_{0}} f, \quad R_{1}^{2}=\frac{9}{4 B_{0} D_{0}}\left[f^{2}-\frac{4}{3}\left(A_{0}-C_{0}\right) f+\frac{2}{9}\left(A_{0}-C_{0}\right)\left(2 A_{0}+C_{0}\right)\right] .
$$

A globally defined solution exists if $R_{1}^{2}$ is positive for all values of $f$. We note that the roots of the polynomial defining $R_{1}^{2}$ are

$$
f_{A, B}=\frac{1}{3}\left[2\left(A_{0}-C_{0}\right) \pm \sqrt{6 C_{0}\left(C_{0}-A_{0}\right)}\right]
$$

Hence, for $C_{0}\left(C_{0}-A_{0}\right)<0$ the function $R_{1}^{2}$ is sign-definite and can be chosen to be positive definite. For $C_{0}\left(C_{0}-A_{0}\right)>0$ a more careful analysis is required. Thus solution (4.5) provides a global solution if the roots satisfy one of the following relations:

$$
\begin{gathered}
f_{A} \leq f_{1} \leq f \leq f_{2} \leq f_{B}, \quad f_{A} \leq f_{B} \leq f_{1} \leq f \leq f_{2} \\
f_{1} \leq f \leq f_{2} \leq f_{A} \leq f_{B}
\end{gathered}
$$

Similarly, case (4.7) and (4.11) both require

$$
f_{A} \leq f_{B} \leq f_{3} \leq f
$$

Multiple roots occur in the two excluded cases $C_{0}=0$ and $C_{0}=A_{0}$, but also in the allowed case

$$
C_{0}=-2 A_{0}
$$

For $A_{0}<0$ we obtain from eq.(4.6)

$$
\begin{gathered}
R_{2}=\frac{6\left|A_{0}\right|}{B_{0}} \frac{1}{\cosh ^{2}\left(\sqrt{\left|A_{0}\right|}\left(\xi-\xi_{0}\right)\right)} \\
R_{1}=\frac{6\left|A_{0}\right|}{\sqrt{-B_{0} D_{0}}} \frac{\sinh \left(\sqrt{\left|A_{0}\right|}\left(\xi-\xi_{0}\right)\right)}{\cosh ^{2}\left(\sqrt{\left|A_{0}\right|}\left(\xi-\xi_{0}\right)\right)}
\end{gathered}
$$


so that we must require $B_{0} D_{0}<0$.

Similarly, for $A_{0}<0$ eq.(4.9) provides the solution

$$
\begin{aligned}
& R_{2}=-\frac{6\left|A_{0}\right|}{B_{0}} \frac{1}{\sinh ^{2}\left(\sqrt{\left|A_{0}\right|}\left(\xi-\xi_{0}\right)\right)} \\
& R_{1}=\frac{6\left|A_{0}\right|}{\sqrt{B_{0} D_{0}}} \frac{\cosh \left(\sqrt{\left|A_{0}\right|}\left(\xi-\xi_{0}\right)\right)}{\sinh ^{2}\left(\sqrt{\left|A_{0}\right|}\left(\xi-\xi_{0}\right)\right)}
\end{aligned}
$$

with the requirement $B_{0} D_{0}>0$.

For $A_{0}>0$ it is solution (4.8) that is relevant and yields:

$$
\begin{gathered}
R_{2}=-\frac{6 A_{0}}{B_{0}} \frac{1}{\sin ^{2}\left(\sqrt{A_{0}}\left(\xi-\xi_{0}\right)\right)} \\
R_{1}=\frac{6 A_{0}}{\sqrt{B_{0} D_{0}}} \frac{\cos \left(\sqrt{A_{0}}\left(\xi-\xi_{0}\right)\right)}{\sin ^{2}\left(\sqrt{A_{0}}\left(\xi-\xi_{0}\right)\right)}, \quad B_{0} D_{0}>0 .
\end{gathered}
$$

2. The case $(3.25): C_{0}=0$

Eq.(4.3) is

$$
f^{\prime 2}=f^{3}-2 A_{0} f^{2}+\frac{4}{3} A_{0}^{2} f+\gamma_{0} \frac{4 B_{0}^{2}}{9}
$$

with $\gamma_{0}$ arbitrary and

$$
R_{2}=-\frac{3}{2 B_{0}} f, \quad R_{1}=\frac{1}{2 \sqrt{B_{0} D_{0}}}\left(-3 f+2 A_{0}\right), \quad B_{0} D_{0}>0
$$

A multiple root, namely a triple one, occurs in one case only

$$
f^{\prime 2}=\left(f-\frac{2}{3} A_{0}\right)^{3}, \quad \gamma_{0}=-\frac{2 A_{0}^{3}}{3 B_{0}^{2}}
$$

and we have

$$
\begin{gathered}
R_{2}=-\frac{1}{B_{0}}\left(\frac{6}{\left(\xi-\xi_{0}\right)^{2}}+A_{0}\right), \\
R_{1}=\frac{-6}{\sqrt{B_{0} D_{0}}} \frac{1}{\left(\xi-\xi_{0}\right)^{2}} .
\end{gathered}
$$


In all other cases two of the roots $f_{i}$ are complex and $f$ is given in eq.(4.11),(4.12).

$$
\text { 3. The case }(3.28): C_{0}=A_{0}
$$

Eq.(4.3) in this case is

$$
f^{\prime 2}=f^{3}-A_{0} f^{2}+\gamma_{0} \frac{4 B_{0}^{2}}{9}
$$

where $\gamma_{0}$ is arbitrary and we have

$$
R_{2}=-\frac{3}{2 B_{0}} f, \quad R_{1}=-\frac{3}{2 \sqrt{B_{0} D_{0}}} f, \quad B_{0} D_{0}>0 .
$$

The discriminant of the cubic equation $f^{\prime 2}=0$ is

$$
D=\frac{16}{9}\left(A_{0}^{3}-3 B_{0}^{2} \gamma_{0}\right) B_{0}^{2} \gamma_{0}
$$

For $D<0$ two roots are complex, for $D>0$ they are all real and distinct, for $D=0$ we have a double or triple root. The absence of a first degree term in (4.26) implies that for $f_{i}$ real the three signs of $f_{i}$ cannot be all the same. Moreover, if a root is equal to $f_{i}=0$, it must be double.

The elementary solutions in this case are:

1. $\gamma_{0}=\frac{A_{0}^{3}}{3 B_{0}^{2}}$. The finite solitary wave, the singular solitary wave, the singular periodic solution and the singular algebraic solutions in this case are:

$$
\begin{gathered}
R_{2}=\sqrt{\frac{D_{0}}{B_{0}}} R_{1}=-\frac{A_{0}}{2 B_{0}}\left[3 \tanh ^{2}\left(\frac{\sqrt{\mathrm{A}_{0}}}{2}\left(\xi-\xi_{0}\right)\right)-1\right], \quad \mathrm{A}_{0}>0 \\
R_{2}=\sqrt{\frac{D_{0}}{B_{0}}} R_{1}=-\frac{A_{0}}{2 B_{0}}\left[3 \operatorname{cotanh}^{2}\left(\frac{\sqrt{\mathrm{A}_{0}}}{2}\left(\xi-\xi_{0}\right)\right)-1\right], \quad \mathrm{A}_{0}>0 \\
R_{2}=\sqrt{\frac{D_{0}}{B_{0}}} R_{1}=-\frac{\left|A_{0}\right|}{2 B_{0}}\left[\frac{3}{\sin ^{2}\left(\frac{\sqrt{\left|A_{0}\right|}}{2}\left(\xi-\xi_{0}\right)\right)}-2\right], \quad A_{0}<0 \\
R_{2}=\sqrt{\frac{D_{0}}{B_{0}}} R_{1}=-\frac{6}{B_{0}\left(\xi-\xi_{0}\right)^{2}}, \quad A_{0}=0
\end{gathered}
$$

respectively. 
2. $\gamma_{0}=0$. The four different real solutions in this case are:

$$
\begin{aligned}
& R_{2}=\frac{3}{2 B_{0}}\left|A_{0}\right| \operatorname{sech}^{2}\left(\frac{\sqrt{\left|\mathrm{A}_{0}\right|}}{2}\left(\xi-\xi_{0}\right)\right), \quad \mathrm{A}_{0}<0 \\
& R_{2}=-\frac{3}{2 B_{0}}\left|A_{0}\right| \frac{1}{\sinh ^{2}\left(\frac{\sqrt{\left|A_{0}\right|}}{2}\left(\xi-\xi_{0}\right)\right)}, \quad A_{0}<0 \\
& R_{2}=-\frac{3 A_{0}}{2 B_{0}} \frac{1}{\sin ^{2}\left(\frac{\sqrt{A_{0}}}{2}\left(\xi-\xi_{0}\right)\right)}, \quad A_{0}>0
\end{aligned}
$$

and for $A_{0}=0$ we reobtain solution (4.31).

\section{Solutions for $\alpha=0$}

Eq.(4.3) (obtained from eq.(3.40)) in this case is

$$
f^{\prime 2}=f^{3}-4 A_{0} f^{2}-2 B_{0} S f-4 M^{2} B_{0}^{2}
$$

where $S$ and $M$ are arbitrary. All possibilities for the three roots $f_{i}$ occur, but we have the restriction

$$
f_{1} f_{2} f_{3} \geq 0
$$

We have

$$
R_{2}=-\frac{1}{2 B_{0}} f, \quad R_{1}=\sqrt{-\epsilon \frac{\left(2 \kappa_{1}+\beta+2 \delta \omega_{1}\right)}{2 B_{0}} f} .
$$

For $R_{1}$ to be globally defined we need $f$ to be sign definite. This imposes the following restrictions on the roots for each of the solutions of section (4.1).

Solutions (4.5) and (4.6):

$$
f_{1} \leq f \leq f_{2} \leq 0 \leq f_{3} \text { or } 0 \leq f_{1} \leq f \leq f_{2} \leq f_{3} .
$$

Solutions (4.7), (4.8) and (4.9):

$$
0 \leq f_{1} \leq f_{2} \leq f_{3} \leq f \text { or } f_{1} \leq f_{2} \leq 0 \leq f_{3} \leq f .
$$

Solution (4.10): 


$$
0 \leq f_{1}=f_{2}=f_{3} \leq f .
$$

Solution (4.11):

$$
0 \leq f_{3} \leq f
$$

\section{OTHER EXPLICIT SOLUTIONS}

In Section 4 we presented solutions of eq.(2.1) satisfying $a_{1} a_{2} \neq 0$ and such that $\left|a_{1}\right|$ and $\left|a_{2}\right|$ are not constant. Let us now discuss these previously rejected solutions.

1. Solutions with $a_{1}=0$

For $\alpha \neq 0$ we put

$$
a_{2}(x, t)=\omega(x, t) \exp i\left[-\left(\beta+\frac{\delta^{2}}{2 \alpha}\right) t-\frac{\delta}{\alpha} x\right] .
$$

The eq.(2.1) for $a_{2}(x, t), a_{1}=0$ reduces to the linear Schrödinger equation

$$
i \omega_{t}-\frac{\alpha}{2} \omega_{x x}=0
$$

For $\alpha=0$ the solution of eq.(2.1) is

$$
a_{2}(x, t)=e^{-i \beta t} \omega(\xi), \quad \xi=x+\delta t
$$

where $\omega(\xi)$ is an arbitrary (complex) function.

2. Solutions with $R_{1}$ and $R_{2}$ constant

We require that $\phi_{1}$ and $\phi_{2}$ should not be constant, otherwise we obtain $a_{1}=a_{2}=0$. Eq.(2.6),..,(2.9) for $R_{1}$ and $R_{2}$ constant imply that we must have $\sin \phi=0$, i.e. the solutions will be phase locked.

Explicitly, for $\alpha \neq 0$ we have 


$$
\begin{gathered}
R_{2}=-\frac{r \omega^{2}}{2 \epsilon}\left(\gamma_{1}^{2}-2 A \gamma_{1}+D\right) \\
R_{1}^{2}=\frac{\alpha r \omega^{4}}{4}\left(4 \gamma_{1}^{2}-2 B \gamma_{1}+C\right)\left(\gamma_{1}^{2}-2 A \gamma_{1}+D\right) \\
\phi_{1}=\gamma_{1} \xi+\gamma_{2} \\
\phi_{2}=2 \gamma_{1} \xi+2 \gamma_{2}+k \pi, \quad \epsilon=(-1)^{k}
\end{gathered}
$$

where $\gamma_{1}$ and $\gamma_{2}$ are arbitrary constants.

For $\alpha=0$ we have $\phi_{1}$ and $\phi_{2}$ as in (5.4)

$$
\begin{gathered}
R_{2}=-\epsilon\left[\frac{r \omega^{2}}{2} \gamma_{1}^{2}+\left(v-r \omega \omega_{1}\right) \gamma_{1}+\frac{r \omega_{1}^{2}-2 \kappa_{1}}{2}\right] \\
R_{1}^{2}=-\epsilon\left[(v+\delta \omega) \gamma_{1}-\left(2 \kappa_{1}+\beta+\delta \omega_{1}\right)\right] R_{2}
\end{gathered}
$$

\section{CONCLUSION}

The symmetries used in this article are only those that exist in the generic case of the cascading equations (2.1), i.e. for all values of the constants $r, \alpha, \beta$ and $\delta$. This "generic" symmetry algebra is summed up in eq.(2.3), representing space and time translations and a shift in the phases of the fundamental and second harmonic waves.

The reduction to the system of ordinary differential equations (2.6)...(2.9) was achieved by requiring that solutions be invariant under the one-dimensional subgroup of the symmetry group, corresponding to the Lie algebra element (2.4). In order to decouple these equations we had to impose either $a_{1}=0$ (no fundamental harmonic), or the supplementary condition (3.1) on the phases (phase locked solutions). The existence of the symmetry $W$ in eq.(2.3) guarantees that if the phases locking condition (3.1) is imposed on the initial conditions, it will survive for all times.

Thus, by construction, all the explicit solutions obtained in this article are phase locked travelling waves. Let us discuss some of their features. 
Solution (4.14), for certain values of the parameters $r, \alpha, \beta, \delta$, characterizing the material involved, and of the constants $\omega, v, \omega_{1}$ and $\kappa_{1}$, characterizing initial conditions, can be periodic finite waves (see eq.(4.5)). The second harmonic $R_{2}$ then oscillates in the interval $\left(-\frac{3}{2 B_{0}} f_{1},-\frac{3}{2 B_{0}} f_{2}\right)$ and the fundamental wave also oscillates between finite limits.

For other conditions (see eq.(4.18)) the elliptic function solutions reduce to solitary waves (4.19) with the second harmonic going through a zero when the fundamental one reaches its maximum value. Asymptotically both waves (4.19) tend to zero with the second harmonic vanishing at a faster rate.

Many of the obtained solutions are singular, either at some specific point $\xi=\xi_{0}$ as in eq.(4.20), or periodically, as in (4.7), or (4.11).

For conditions leading to eq.(3.25) the second harmonic differs from the fundamental one just by a proportionality factor and a constant shift of the amplitude (see eq.(3.26)). Similarly, for conditions (3.28), the two waves are simply proportional.

Singular solutions coexist with the finite ones for all the values of the coefficients in eq.(2.1). Their physical meaning needs a separate investigation: they may be an indication that higher harmonics or dissipative effects were unjustifiably ignored in the derivation of these equations. These effects would tend to smoothen out the singularities and possibly turn them into finite resonance phenomena.

The existence of families of elliptic function solutions can be viewed as a manifestation of "partial integrability". We have shown that the studied equations do not, in general have the Painlevé property. For special values of the constants involved we do get solutions that do have this property: they have no movable singularities other than poles.

Finally let us mention that for particular values of the constants in eq.(2.1) the symmetry group may be larger. For instance, for $\beta=\delta=0$ the equations are invariant under dilatations generated by:

$$
D=x \partial_{x}+2 t \partial_{t}-2\left(R_{1} \partial_{R_{1}}+R_{2} \partial_{R_{2}}\right)
$$


This raises the posibility of obtaining self-similar solutions of the form

$$
\begin{gathered}
a_{1}(\xi)=\frac{1}{t} F_{1}(\xi) e^{i \phi_{1}(\xi)} e^{\frac{i}{2} \ln t}, \\
a_{2}(\xi)=\frac{1}{t} F_{2}(\xi) e^{i \phi_{2}(\xi)} e^{i \ln t} \\
\xi=\frac{x}{\sqrt{t}} .
\end{gathered}
$$

Since self-similar solutions are particularly important (and stable) in optical systems with memory [20,21,22], this situation may be will worth exploring.

\section{ACKNOWLEDGEMENTS}

The authors thank D.Levi, Z.Thomova and Yu.S.Kivshar for helpful discussions. S. L. acknowledges a scholarship from NSERC (National Science and Engineering Research Council of Canada) for his Ph.D studies. The research of P.W. was partly supported by research grants from NSERC of Canada and FCAR du Québec.

[1] See e.g., G.P. Agrawal, Nonlinear Fiber Optics, Academic, San Diego (1989), Chap. 2.

[2] W.E.Torruellas, Z.Wang, D.J.Hagan, E.W.Van Strykland, G.I.Stegeman, L.Torner and C.R.Menyuk, Phys.Rev.E 74, 5036 (1995).

[3] C.R.Menyuk, R.Schiek and L.Torner Journ.Opt.Soc.Am.B 11, 2434-2443 (1994).

[4] L.Torner, C.R.Menyuk, W.E.Torruellas and G.I. Stegeman, Opt.Lett. 20, 13 (1995).

[5] A.V.Buryak and Yu.S.Kivshar, Opt.Lett.19, 1612-1614 (1994).

[6] M.J.Werner and P.D.Drummond, Journ.Opt.Soc.Am.B 10,2390-2393 (1993).

[7] M.J.Werner and P.D.Drummond, Opt.Lett. 19, 613-615 (1994).

[8] P.Ferro and S.Trillo, Phys.Rev.E 51, 4994-4998 (1995). 
[9] G.I.Stegeman, M.Sheik-Bhae, E.Van Strykland and G.Assanto, Opt.Lett. 18, 13-15 (1993).

[10] A.V.Buryak and Yu.S.Kivshar, Phys.Lett.A 197 407-412 (1995).

[11] A.V.Buryak and Yu.S.Kivshar, Phys.Rev.A 51 R41-R44 (1995).

[12] A.V.Buryak and Yu.S.Kivshar, Opt.Lett.20, 834-836 (1995).

[13] M.J.Ablowitz, A.Ramani and H.Segur, Journ.Math.Phys. 21, 715 (1980).

[14] M.J.Ablowitz and H.Segur, Solitons and the Inverse Scattering Transform, SIAM, Philadelphia, (1981).

[15] P.J.Olver, Applications of Lie Groups to Differential Equations, Springer, Berlin (1986).

[16] B.Champagne, W.Hereman and P.Winternitz, Comput.Phys.Commun. 66, 319 (1991).

[17] P.Winternitz, Lie Groups and Solutions of Nonlinear Partial Differential Equations, in: Integrable Systems, Quantum Groups and Quantum Field Theory, Kluwer, Dordrecht (1993).

[18] D.Rand and P.Winternitz, Comput.Phys.Commun. 42, 359 (1986).

[19] P.F.Byrd and M.D.Friedmann, Handbook of Elliptic Integrals for Engineers and Scientists, Springer, New York (1971).

[20] D.Levi, C.R.Menyuk and P.Winternitz, Phys.Rev.A 44, 6057 (1991); 49, 2844 (1994).

[21] C.R.Menyuk, D.Levi and P.Winternitz, Phys.Rev.Lett. 69, 3048 (1992).

[22] D.Levi, C.R.Menyuk and P.Winternitz, (editors). Self-Similarity in Stimulated Raman Scattering (Publications CRM, Montréal, 1994). 